\title{
OFFSET CALCULATION FOR TRAFFIC SCENARIOS
}

\author{
Andreas Volkert, Niklas Peinecke \\ Institute of Flight Guidance \\ DLR (German Aerospace Center)
}

\author{
Braunschweig, Germany
}

\begin{abstract}
The integration of unmanned aerial systems (UAS) and remotely piloted aircraft systems (RPAS) will play a key-role in the world-wide aviation for the next years. In order to safely integrate UAS in the existing manned aviation, they have to follow the same rules and commands as manned aviation currently does. One of the major challenges is to carry out proper detect and avoid (DAA) with such vehicles. A proper working DAA is essential in certain airspaces where separation from other airspace users is not provided by ATC. To ensure a safe detection one possibility is to equip the vehicle with active sensors that can sense surrounding traffic. To evaluate the level of safety, an understanding for minimal detection ranges of such systems has to be established.
\end{abstract}

Where ATC is not responsible for separation, pilots are responsible to stay "well-clear" from each other. Manned aviation works with such an imprecise rule, but a DAA system needs exact numbers for minimum separation distances in order to "remainwell-clear". The numerical approach in this paper shows one possibility to calculate the offset of trajectories in different representative traffic scenarios. The offset shall be of a size to just not trigger TCAS (Traffic Collision Avoidance System) RA (Resolution Advisory) alerts, but can be used to evaluate DAA algorithms.

The number of traffic scenarios defined for this paper aim at covering most cases encountered in practice. Thus, the simulation scenarios constructed from these principles can be used to determine minimal sensor detection ranges that a real-world system has to adhere to in order to be considered safe in mixed-traffic operations.

\section{Introduction}

With more and more parties taking an interest in commercial use of unmanned and remotely piloted aircraft systems (RPAS) it becomes necessary to integrate these vehicles into the traditional airspace. Current airspace classification by its primary use reflects the main focus of traditional air traffic management (ATM) on the upper airspace. For example, the German airspace comprises of classes $\mathrm{C}, \mathrm{D}, \mathrm{E}$ and $\mathrm{G}$, with almost the entire airspace below $2500 \mathrm{ft}$ above ground level (AGL) belonging to class G. Unmanned aerial vehicles are not directly covered by the regulations that apply to airspace $\mathrm{G}$.

Consequently, stakeholders have stated an interest to regulate and possibly restructure parts of the airspace to meet the requirements of unmanned aerial systems (UAS). In the US the concept of an UAS air traffic management (UTM) has been devised, and is currently discussed and developed further [1]. In Europe a similar concept named $U$ Space has been evolved. The concept itself is targeted but not limited to urban airspace: The Warsaw Declaration [2] states that there is a need for "the development of the concept of the U-Space on access to low level airspace, especially in urban areas." This makes it clear that currently a demand is seen to solve the problem of integrating UAS into airspace.

SESAR's recent study "European Drones Outlook Study - Unlocking the value of Europe" predicts a European fleet of over 8 million drones by 2050. Among these more than 500,000 drones will be used for commercial applications [3]. The aforementioned UTM concepts - NASA UTM and $U$-space - provide infrastructure and some concepts for operating the airspace free of conflicts. DAA plays a central role in each of these concepts.

In case of mixed traffic, for example near an airport, UAS have to be integrated in current airspace and have to share it with manned aviation. Present flight rules shall be adopted and obeyed. Aircraft have to be separated in their airspace to avoid near misses or even mid-air incidents. In European airspace classes A to $\mathrm{F}$ separation is provided by ATC [4]. Nevertheless, ATC can command to carry 
out self-separation in which case the UAS would need DAA equipment. In class $G$ separation is not provided by ATC, pilots are responsible to stay wellclear on their own. Again, some means of DAA are needed.

\section{Remain Well-Clear}

Manned aviation follows the rules of the air which are written down in ICAO Annex 2 [18] and recently transferred to SERA Part A (Standardized European Rules of the Air) [19]. The notion "wellclear" is used in ICAO Annex 2 but neither provides any minimum separation distances nor explains what remain-well-clear exactly is. This leaves room for interpretation of a safe distance to other aircraft.

The ICAO Annex 2 relies on the human eye and gut-feeling of the pilot to estimate a safe separation in order to stay well-clear throughout the whole maneuver. A UAS on the other hand, as autonomous or at least partly automated machinery needs proper definitions of Remain-Well-Clear in order to operate safely. When implementing UAS into civil airspace, exact well-clear distances have to be determined. The Offset (further discussed on page 4) is an important input for the scenarios, which will be treated in a latter section of this paper. Generally speaking, the more complex the traffic scenario is, the more complex the Remain-Well-Clear situation. The Offset adds complexity to the traffic scenarios, since it changes the encounter geometries. Evasive manoeuvers may lead to even more dangerous situations. The Offset changes the relative bearing and relative speed over time, even if both aircraft (as assumed) remain at a constant speed and heading. So the Offset has an essential consequence on the complexity of Remain-Well-Clear and is therefore important to consider.

\section{Sensor detection range}

In order to make UAS "see" other air traffic, sensors have to be installed that are able to detect air traffic. Different technologies could provide the UAS with distance detections. To choose an appropriate sensor technique the required sensor detection range has to be determined. This sensor detection range is also known as radar declaration range (RDR) [14]. But we use the term sensor detection range since other sensors than radar are common in the context of UAS. In this paper we develop scenarios for determining these sensor ranges. Depending on the traffic scenario, the required detection range varies. Consequently, a number of different traffic scenarios is developed that can be used in simulations to determine sensor ranges. The results in turn can then be used to give recommendations about necessary detection ranges.

\section{Related Work}

The integration of unmanned or remotely piloted aircraft has been investigated for several years now [5], and various concepts for different use-cases and scenarios have been suggested. Simulations have shown that in principal an integration of single UAS into the existing ATM system is possible [6]. Other concepts aim at changing the airspace structure specifically for integrating small UAS, for example by introducing specialized UAS traffic management (UTM). A popular example is NASA UTM [1]. To advance the European counterpart $U$-Space recently a blue-print paper has been released [7]. Similarly, the Global UTM Association has drafted a holistic architecture for a UTM implementation covering aspects of $U$-space [8].

Simulations have been devised to demonstrate different UTM concepts. Ramasamy et al. have shown a separation concept in MATLAB-based simulations that includes the avoidance of individual buildings [9]. Simulations have been carried out to determine the possibility of operating drone networks in urban areas [10]. Further, integration concepts have been proposed for the non-urban airspace [11].

Mũnoz, C. et. al. published a paper with the title "A TCAS-II Resolution Advisory Detection Algorithm" [12]. That paper has the same objective like this paper and shall therefore be briefly discussed. Mũnoz' paper aims for an algorithm to predict the detection of a TCAS corrective Resolution Advisory. The approach is formally verified to characterize all encounter geometries between two aircraft (that lead to a resolution advisory within a given look-ahead time interval) completely and correctly. The author of this paper found the analytical approach too complex and thus developed a numerical approach. Nevertheless, both papers share the same assumptions:

- Accurate vector state information for two aircraft, 
- Aircraft linear trajectories,

- Constant speed of both aircraft and

- TCAS sensitivity level remains constant

The numeric approach uses easy mathematical terms and is therefore easy to implement in an engineering context. It also provides data for the steps in-between and can show exactly when and how long a Resolution Advisory is triggered. However, a comparison of results was not performed.

In the following year Mũnoz, C. et. al. have continued their work on DAA problems. Their notion of Well-Clear, together with a prototype DAA implementation has been demonstrated in the DAIDALUS system [13]. Consequently, their work resulted in an RTCA standard, the DO-365 [14]. This document also includes an extended formal definition of Well-Clear that can be adapted to TCAS and similar systems.

\section{Traffic Scenarios}

The sensor detection range is defined as the largest distance between two aircraft at which a safe detection can be achieved. This detection range depends on multiple parameters. For example, the detection range between two aircraft may not be symmetric: In case one aircraft is rather large while the other is small (drones may even be tiny) the detection range from the large to the small aircraft might be considerable larger than vice versa, given that both use the same sensor. Further, the surroundings of an aircraft can degrade its detectability by sensors. For instance, a low-flying UAS in an urban area can become almost invisible from above due to optical effects, multi-path phenomena, etc., while the UAS itself can clearly detect traffic above against the blue sky. Clearly, many more of such parameters exist, and most of them are depending on the actual use-case scenario.

\section{Speed and Geometry}

In this paper we only address parameters that are independent of the actual use-case and of the vehicle used. These parameters are the speeds of the aircraft, and the overall encounter geometry. It is assumed that the manned aircraft has always right of way so the UAS has to give way, according to the rules of the air. Obeying the rules of the air means that the UAS has to make an evasive maneuver to the right if they are flying head-on or approximately headon [15]. We assume that a right turn is preferred over a left turn with both aircraft on a converging course, even though the rules of the air do not specify the direction of turn. In this paper changes of altitude or speed as means of an evasive maneuver are not considered.

Simulations will be performed, calculating the required evasive maneuver and the maximum required detection range of the sensors. The description of simulations and their outcome will not be detailed in this paper. This paper focusses on the description of the numerical approach to determine sensor detection ranges. Triggering a TCAS resolution advisory (RA) at the other aircraft must be avoided and is an essential requirement for the simulations and for the traffic scenarios. Triggering TCAS traffic alerts (TA) at the other aircraft while executing an evasive maneuver is allowed though.

Traffic scenarios were created with respect to the mentioned constraints. The speeds of the other aircraft as well as the speed of the UAS vary between $0 \mathrm{kts}$ and 500kts in equal intervals. Looking from the other aircraft's perspective, the relative bearing between both aircraft varies from $70^{\circ}$ to $180^{\circ}$, where $180^{\circ}$ is a head-on situation and a relative bearing of $90^{\circ}$ means the UAS approaches from the left.

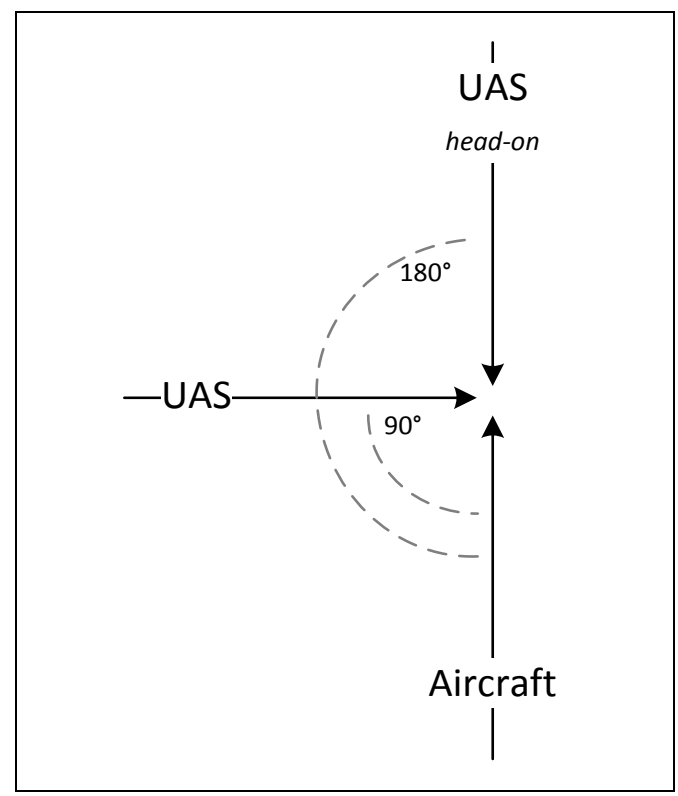

Figure 1. UAS head-on and UAS converging from the left $\left(90^{\circ}\right)$ 
TCAS has different sensitivity levels, which vary with altitude [16]. Each sensitivity level has a different tau value given in units of seconds, refer to Figure 2. The aircraft distance divided by the relative closing speed is a time value given in seconds. This time value is the remaining time until arriving at the target point, thus it shall be called $t_{T P}$. In TCAS II systems $t_{T P}$ is the aforementioned tau value and is used as an estimate for the time until CPA (closest point of approach) although this is only accurate in case the CPA is a real collision point, that is where Offset $=0$.

Is the calculated $t_{T P}$ smaller than the TCAS RA tau value, a resolution advisory is triggered. This was also considered when traffic scenarios were created.

Also the DMOD (Distance Modification) value of TCAS II was considered, but never influenced the offset calculation. Hence it is not shown in Figure 2.

\begin{tabular}{|c|c|c|c|}
\hline Own Altitude (feet) & \multirow{2}{*}{ SL } & \multicolumn{2}{|c|}{ Tau (Seconds) } \\
\cline { 3 - 4 } & & TA & RA \\
\hline$<1000($ AGL) & 2 & 20 & N/A \\
\hline $1000-2350($ AGL) & 3 & 25 & 15 \\
\hline $2350-5000$ & 4 & 30 & 20 \\
\hline $5000-10000$ & 5 & 40 & 25 \\
\hline $10000-20000$ & 6 & 45 & 30 \\
\hline $20000-42000$ & 7 & 48 & 35 \\
\hline$>42000$ & 7 & 48 & 35 \\
\hline
\end{tabular}

Figure 2. TCAS definition of sensitivity levels (SL) and tau values [16]

\section{Offset}

The traffic scenarios created so far all describe two aircraft on collision course. In reality this is a possible but rare scenario, and should not be the only type of scenario to investigate. The most common case would be that conflicting aircraft approach each other up to a certain CPA where the distance is below some required safety separation distance. Often the trajectories of aircraft cross each other, with the aircraft arriving at the crossing point at different times. A further case is a head-on scenario (relative bearing $180^{\circ}$ ), where the two trajectories are parallel to each other with a certain distance below safety separation. We refer to this distance as the Offset.

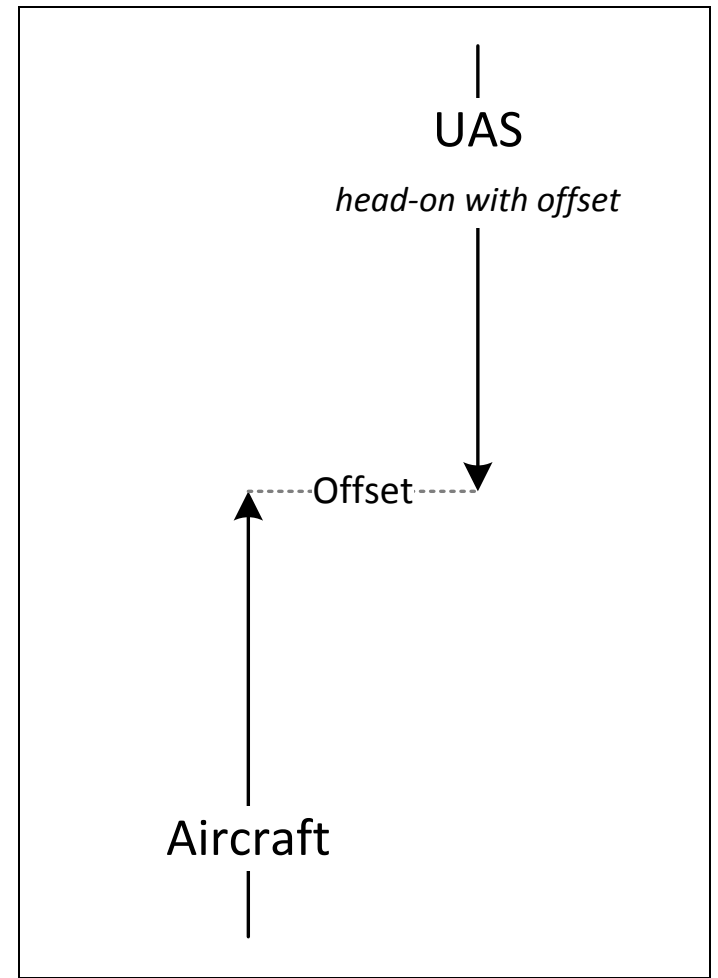

Figure 3. Head-on scenario with an offset to the
left

However, in a head-on scenario with an Offset as shown in Figure 3 the evasive maneuver must start earlier (compared to a head-on scenario with no offset) since only right turns are allowed. This also increases the required detection range of the sensors. For a head-on scenario it is obvious, that the highest required detection range is gained by taking maximum speeds of both aircraft. The offset also increases the detection range, but only up to the point where the offset becomes larger than the safety separation. Then, the TCAS resolution advisory of the other aircraft is not triggered any more. Traffic scenarios where the TCAS RA is not triggered shall not be considered in the simulations.

The maximum applicable offset has to be determined which will just trigger a TCAS resolution advisory if both aircraft would stay on their trajectories. In the simulation this will result in the highest required detection range.

\section{Calculation of Offset}

Microsoft Excel was chosen for development of a calculation model. The model calculates the starting points of both aircraft by the inputs given to the calculation model. These are: 
- Target point,

- Speed of each aircraft: $s_{U}$ and $s_{A}$,

- Relative bearing $\alpha$ and

- Offset $r$

Starting points as well as the target point are given in geographic coordinates. The other aircraft always aims to the given target point. The UAS however only aims for the same target point if Offset $=0$. If the offset has a positive or negative value other than " 0 ", the UAS' target point is shifted on an orthogonal line to the own trajectory. The given offset is the distance along the orthogonal line between the two target points. The specific time to reach the target point can then be calculated from the parameters given.

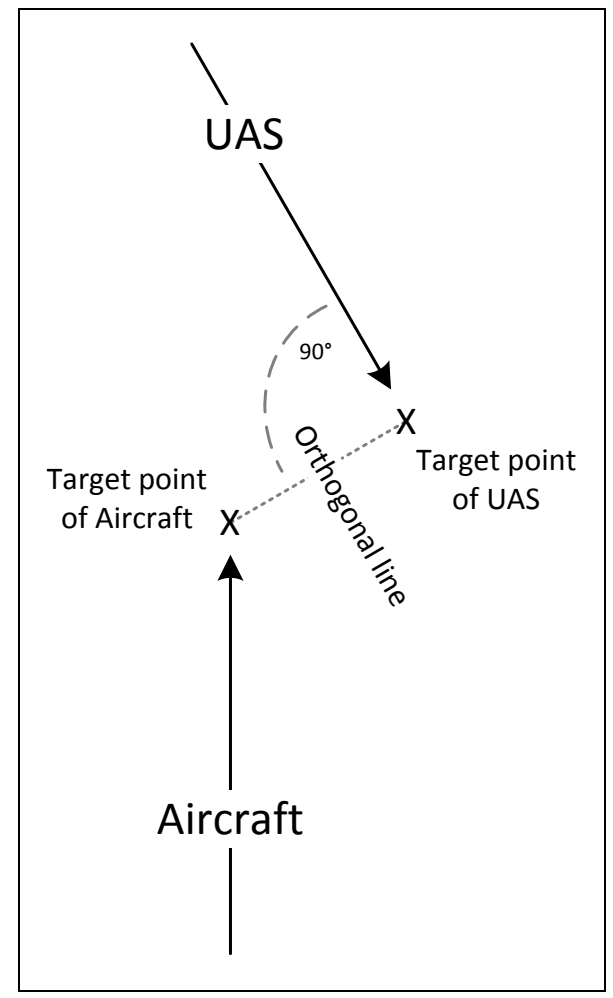

Figure 4. Offset movement on an orthogonal line

Given that the relative bearing is always between $90^{\circ}$ and $270^{\circ}$ this means that the target points are effectively identical to the CPA. This would not be the case for bearings smaller than $90^{\circ}$ or larger than $270^{\circ}$.
Based on the starting points, trajectories and speeds, the distance $d(t)$ and the relative closing speed $\dot{d}(t)$ can be calculated as functions over $t$ :

$$
\begin{gathered}
d(t)=\sqrt{\left(\begin{array}{c}
r \cos \alpha-s_{U} t \sin \alpha \\
s_{A} t-r \sin \alpha-s_{U} t \cos \alpha
\end{array}\right)^{2}} \\
\dot{d}(t)=\frac{\left.t\left(s_{U}{ }^{2}+s_{A}{ }^{2}-2 s_{A} s_{U} \cos \alpha\right)-2 r s_{A} \sin \alpha\right)}{d(t)}
\end{gathered}
$$

The relative closing speed $\dot{d}(t)$ is constant if Offset $=0$. If the offset deviates from " 0 ", the relative closing speed $\dot{d}(t)$ is changing over time.

$$
t_{T P}=\frac{d(t)}{\dot{d}(t)}
$$

$t_{T P}$ is compared to the TCAS resolution advisory tau values. If $t_{T P}$ is positive but smaller than the TCAS tau value, a TCAS RA will be triggered. In traffic scenarios where $t_{T P}$ is always greater than the TCAS RA tau value the results are not of interest for this study, since this traffic scenario would not cause an avoidance maneuver.

Due to the singularity around $t=0$ the graphs of $t_{T P}$ have hyperbolic form. Thus, in order to get sufficient accuracy in plots and numerical evaluations time steps need to become smaller with $t_{T P}$ approaching 0 . For example if $t_{T P}$ is 120 seconds, the time step should be 10 to 15 seconds. If $t_{T P}$ is less than 20 seconds, the time step is chosen as 1 second. The time step gradually decreases with decreasing $t_{T P}$.

Figure 5 and Figure 6 show exemplary plots for two different offsets. The plots have to be interpreted from right to left. The right side of the graphs shows the starting positions of both aircraft, having the biggest distance between them. As the aircraft positions converge $t_{T P}$ comes closer to the TCAS RA tau value. The $t_{T P}$ graph goes sharply upwards with the closing speed approaching 0 and the quotient diverging. The blue graph shows the change of $t_{T P}$ over time, the red graph represents the constant TCAS RA tau value. Therefore the latter is represented by a horizontal line in the plot. If the $t_{T P}$ plot crosses or touches the TCAS RA plot TCAS is triggered, as shown in Figure 5 


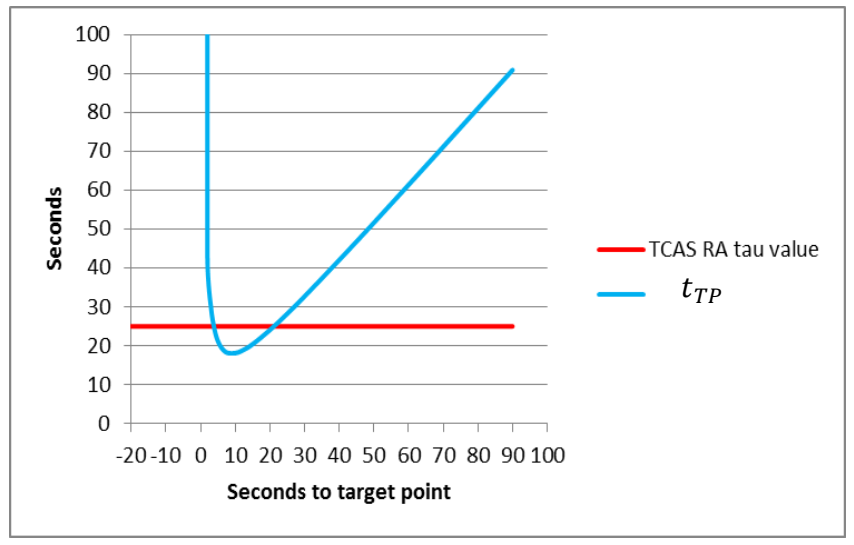

Figure 5. Graphs for TCAS RA tau value and $t_{T P}$, TCAS triggers

In order to find the biggest possible offset the TCAS RA graph must be the tangent of the $t_{T P}$ plot, see Figure 6. This is the case if the derivative of $t_{T P}$ becomes 0 , that is:

$$
\frac{\partial}{\partial t} t_{T P}=\frac{\partial}{\partial t} \frac{d(t)}{\dot{d}(t)}=0
$$

This can be transformed to:

$\frac{\left(s_{A}{ }^{2}-2 s_{A} s_{U} \cos \alpha+s_{U}{ }^{2}\right)\left(t^{2}\left(s_{A}{ }^{2}-2 s_{A} s_{U} \cos \alpha+s_{U}{ }^{2}\right)+r^{2}-2 s_{A} r t \sin \alpha\right)}{\left(-t\left(s_{A}{ }^{2}+s_{U}{ }^{2}\right)+s_{A} r \sin \alpha+2 s_{A} t s_{U} \cos \alpha\right)^{2}}$

$$
=2
$$

This equation has two solutions only one of them is positive. It represents the time $t_{T P \min }$ at which $t_{T P}$ becomes minimal. The offset can be calculated by solving $t_{T P}\left(t_{T P \text { min }}\right)=\tau$ with $\tau$ being the TCAS RA tau value. The easiest way to solve this is by numerical methods. For our purposes an approximate solution ( \pm 1 second $)$ is sufficient, because the Offset we want to calculate does not need to be too accurate. Thus we implemented a graphical solution using the plot graphs shown.

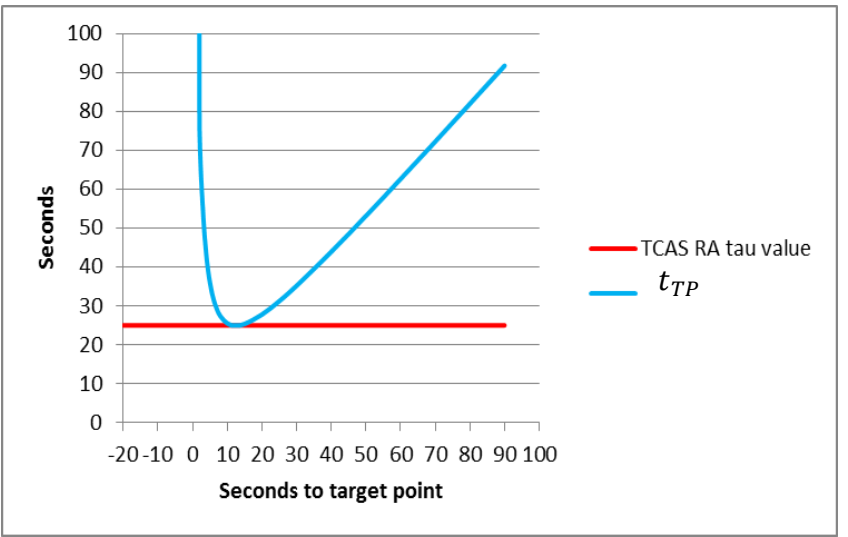

Figure 6. TCAS just triggers, Offset is at its maximum

The calculation can be done with Microsoft Excel. The process of finding the right value for the offset is then a matter of varying the offset until tangential behavior is reached. For each scenario two offsets are required; one offset to the left and one to the right. A macro was written to let Excel iterate the required offsets for each scenario.

\section{Examples of Offsets}

In order to give an impression of the generated offsets the table below will show a couple of representative scenarios. A Scenario ID was defined for a better overview. The first number in the ID represents the Sensitivity Level from TCAS, refer to Figure 2. The second and third number gives the speed in knots of the intruder and the UAS. The forth number shows the relative bearing between both trajectories. The latter states to which side the trajectories are offset; in a head-on scenario L means that the UAS is passing the other aircraft on the left side. In a $90^{\circ}$ scenario L means that the UAS would pass the crossing point of both trajectories first. Figure 3 and Figure 4 show an exemplary offset to the left (L). 


\begin{tabular}{|c|c|c|}
\hline$\#$ & Scenario ID & Offset [NM] \\
\hline I & $7-500-500-180-\mathrm{L}$ & 4,86 \\
\hline II & $5-500-500-180-\mathrm{L}$ & 3,46 \\
\hline III & $7-250-250-180-\mathrm{L}$ & 2,40 \\
\hline IV & $5-250-250-180-\mathrm{L}$ & 1,73 \\
\hline V & $3-250-250-180-\mathrm{L}$ & 1,03 \\
\hline VI & $7-500-500-70-\mathrm{L}$ & 4,56 \\
\hline VII & $7-500-500-70-\mathrm{R}$ & 5,61 \\
\hline VIII & $7-500-500-90-\mathrm{L}$ & 4,86 \\
\hline IX & $7-500-500-90-\mathrm{R}$ & 4,86 \\
\hline X & $5-500-500-70-\mathrm{L}$ & 3,26 \\
\hline XI & $5-500-500-70-\mathrm{R}$ & 4,08 \\
\hline XII & $3-250-250-90-\mathrm{R}$ & 2,42 \\
\hline XIII & $3-100-100-90-\mathrm{R}$ & 0,96 \\
\hline XIV & $3-100-100-70-\mathrm{R}$ & 0,97 \\
\hline
\end{tabular}

Table 1. Examples of Offsets

\section{Discussion}

Looking at the scenarios and their offsets in the previous section a few peculiarities shall be discussed.

Scenarios I and II share the same encounter geometry and the same speeds but have different TCAS Sensitivity Levels. Thus the TCAS tau value is different. The different offsets therefore result from TCAS' characteristics. Figure 7 shows Scenario I.

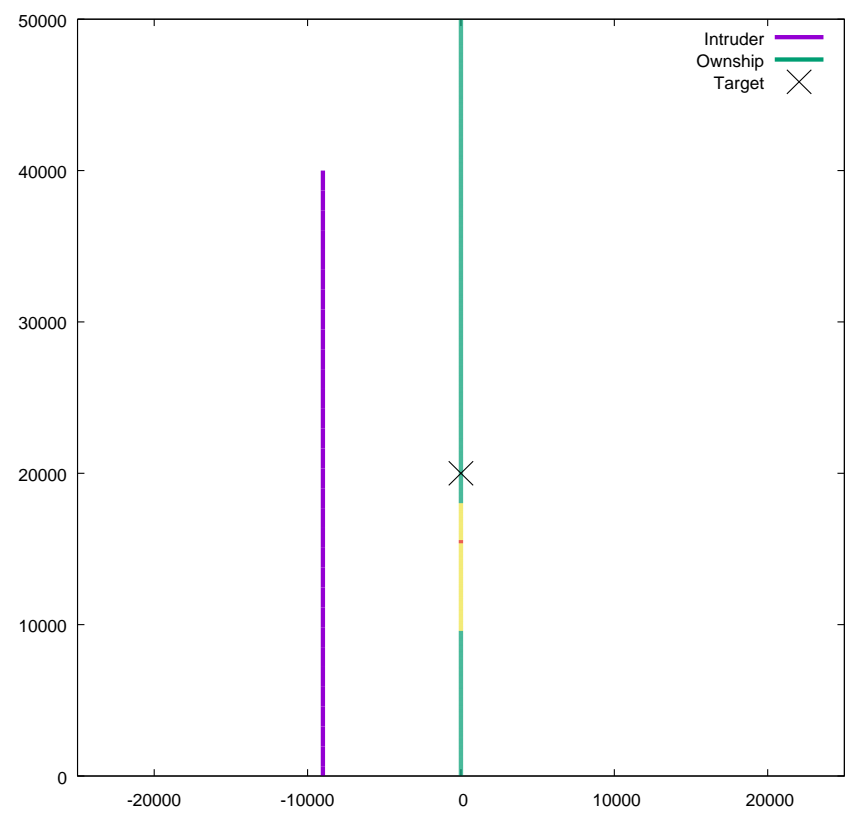

Figure 7. Scenario I
The intruder's path is shown in purple and the ownship's path is shown in green. Yellow represents a TCAS TA, red depicts TCAS RA. The cross shows the target point of the ownship. Both axes show figures in meter. The origin of the plots is always the start point of the ownship.

Keeping the same encounter geometries with halved speeds like in scenarios I and III or II and IV results in a reduction of offsets by about one half. For head-on scenarios like I, II, III and IV the offset is therefore linearly related to the change of relative speed. However, this linearity does not hold for encounter geometries other than head-on $\left(180^{\circ}\right)$.

Keeping the speed steady but changing the encounter geometry from $180^{\circ}$ to $90^{\circ}$ like in scenarios I and VIII, or I and IX, the offset is roughly the same. The plots of the Scenario I (Figure 7), VIII (Figure 8) and IX (Figure 9) show a small red line which indicates that the TCAS RA is just triggered.

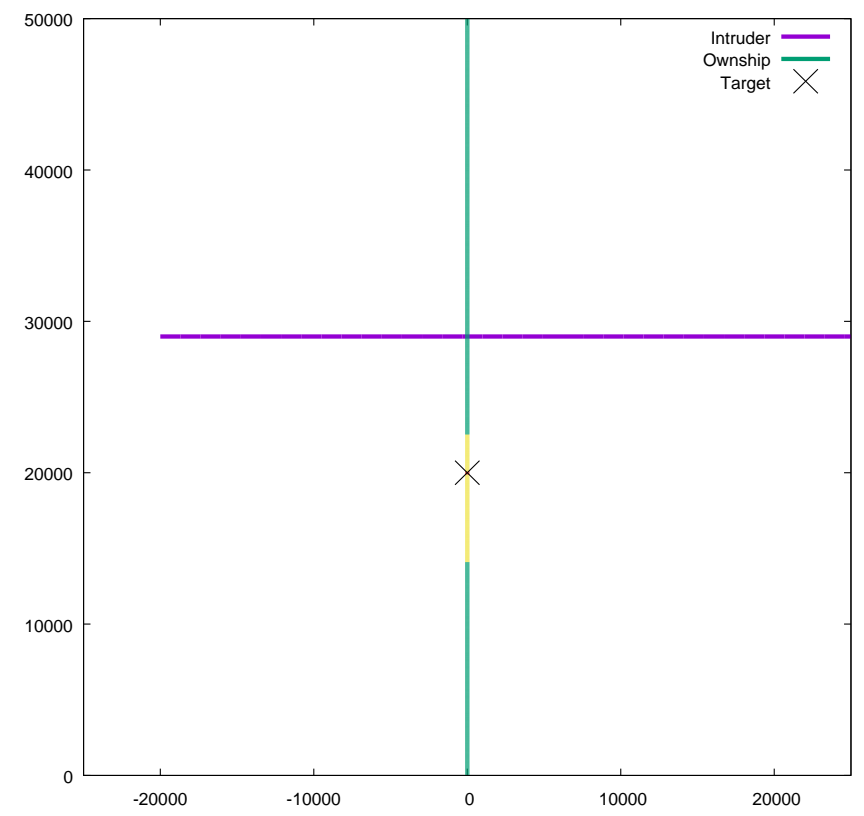

Figure 8. Scenario VIII 


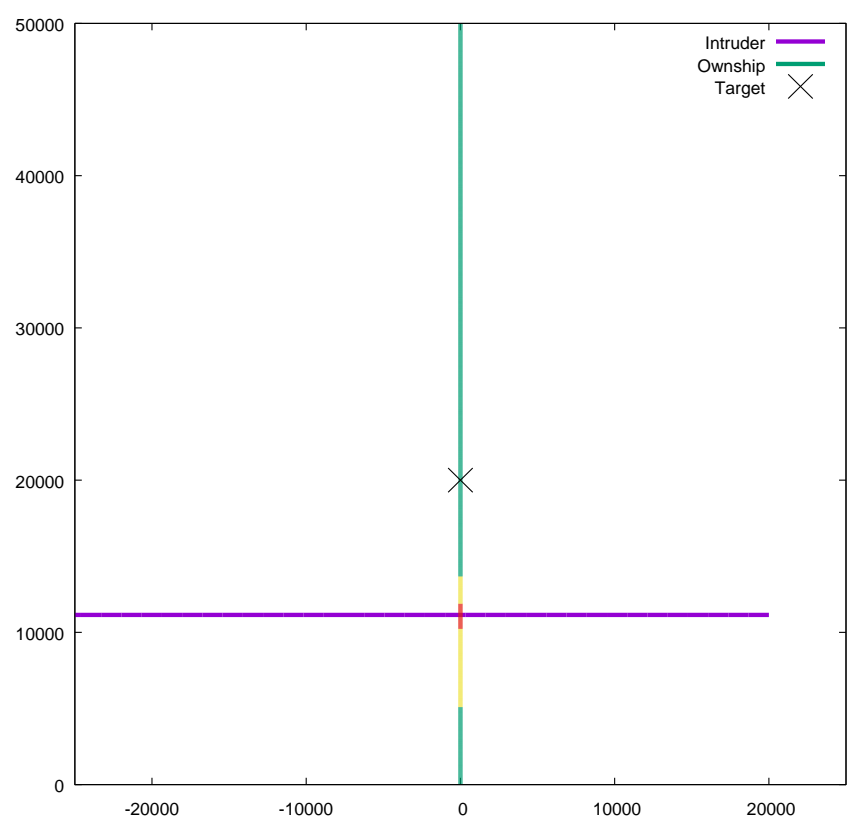

Figure 9. Scenario IX

Scenarios VII stands out as it features the highest offset. This is because the trajectories cross each other before the target point, and the initial relative distance is smaller than in any other scenario with speeds of $500 \mathrm{kts}$ for both aircraft. Thus TCAS is triggered very early.

Comparing Scenario VII and IX the offsets become bigger as the relative bearing gets smaller. This is just true to a certain degree, since a very small relative bearing would result in a very small relative speed and therefore decreases the offset again. So there must be a certain relative bearing where a maximum is reached for the offset. However, this calculation is not part of this paper.

Scenarios V, XIII and XIV feature low altitudes (1000 to $2350 \mathrm{ft}$ AGL [16]) at low speeds. Regarding an offset to the right and comparing scenarios I, VII and IX, encounter geometries of $70^{\circ}$ results in the biggest offsets. This is also true for scenarios XIII and XIV. The range of FLARM is typically $3-5 \mathrm{~km}$ [17], depending upon antenna and installation. That is a range of 1,62 to 2,70 NM. Still scenarios XIV and $\mathrm{XV}$ are well in this range.

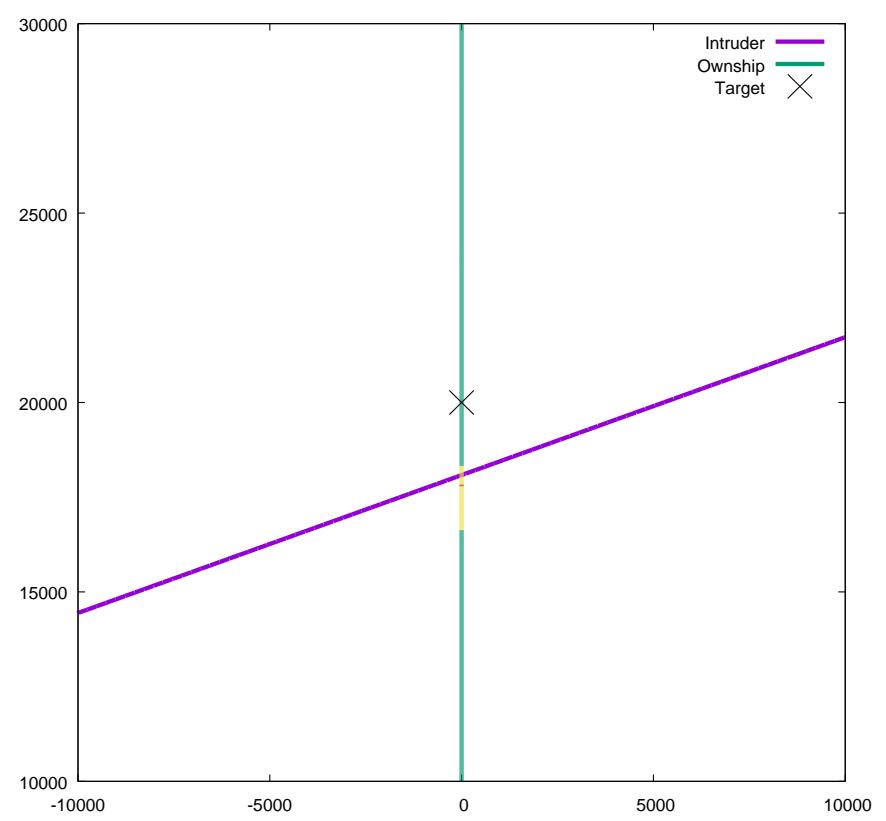

Figure 10. Scenario XIV

\section{Outlook}

The produced scenarios with their offsets will be used to simulate evasive maneuvers of the UAS in order to not trigger TCAS. The focus of the simulation is on the moment of initiation of the evasive maneuver. The $t_{T P}$ from that point in time can be derived as a distance. This distance will be the basis for future calculations of the required minimum sensor detection range. Further work will be done to investigate additional scenarios and conduct simulations studies. The simulations and analyses of the results of the simulations are still pending and will be conducted in a follow-up paper.

\section{References}

[1] Kopardekar, P. H., 2014, "Unmanned aerial system (UAS) traffic management (UTM): Enabling low-altitude airspace and UAS operations", NASA Tech. Rep.

[2] "Warsaw Declaration: Drones as a leverage for jobs and new business opportunities", 2016, High Level Conference on Drones, Warsaw, Poland, [https://www.easa.europa.eu/system/files/dfu/Warsa w\%20Declaration\%20on\%20Drones_24\%20Nov\%2 02016_final_EN.PDF]. 
[3] SESAR Joint Undertaking, 2016, "European Drones Outlook Study: Unlocking the value for Europe".

[4] International Civil Aviation Organization, 2001, ICAO Annex 11, In: ICAO (ed.) 13 ed.

[5] Dalamagkidis, K., K. P. Valavanis, L. A. Piegl, 2008, "On unmanned aircraft systems issues, challenges and operational restrictions preventing integration into the national airspace system." Progress in Aerospace Sciences 44.7, 503-519.

[6] Geister, Dagi, Bernd Korn, Sebastian Tittel, Christiane Edinger, 2013, "Operational integration of UAS into the ATM system", AIAA Infotech@Aerospace (I@A) Conference, Boston, MA.

[7] SESAR Joint Undertaking, 2017, "U-Space Blueprint,"

[https://www.sesarju.eu/sites/default/files/documents/ reports/U-space\%20Blueprint.pdf].

[8] Global UTM Association, 2017, "UAS Traffic Management Architecture," [https://utm.aero/].

[9] Ramasamy, S., R. Sabatini, A. Gardi, 2017, "A Unified Approach to Separation Assurance and Collision Avoidance for UAS Operations and Traffic Management", International Conference on Unmanned Aircraft Systems (ICUAS 2017), Miami, FL, USA.

[10] Peinecke, Niklas, Alexander Kuenz, 2017, "Deconflicting the Urban Drone Airspace." In: Proceedings of the 36th DASC. DASC 2017, 17.-21. Sep. 2017, St. Petersburg, FL, USA.

[11] Peinecke, Niklas, Andreas Volkert, Bernd Korn, 2017, "Minimum Risk Low Altitude Airspace Integration for Larger Cargo UAS." In: Proceedings of the ICNS 2017. IEEE Press. Integrated
Communications Navigation and Surveillance Conference (ICNS 2017), 18.-20. April 2017, Washington DC, USA.

[12] Mũnoz, C., A. Narkawicz, J. Chamberlain, "A TCAS-II Resolution Advisory Detection Algorithm" [https://shemesh.larc.nasa.gov/people/cam/publicatio ns/gnc2013-draft.pdf].

[13] C. Muñoz et al., "DAIDALUS: Detect and Avoid Alerting Logic for Unmanned Systems," 2015 IEEE/AIAA 34th Digital Avionics Systems Conference (DASC), Prague, 2015, pp. 5A1-1-5A112.

[14] RTCA DO-365: Minimum Operational Performance Standards (MOPS) for Detect and Avoid (DAA) Systems, 2017 Edition, May 31, 2017

[15] International Civil Aviation Organization, 2012, Commission Implementing Regulation (EU) No 923/2012. In: ICAO (ed.).

[16] Federal Aviation Administration, 2011, Introduction to TCAS II Version 7.1. In: Transportation, U. S. D. O. (ed.).

[17] FLARM Technology Ltd., 2016, Installation Manual FLARM Collision Avoidance System. Retrieved from Baar-Switzerland.

[18] International Civil Aviation Organization, 2005, ICAO Annex 2, In: ICAO (ed.) 10 ed.

[19] European Commission, 2009, Mandate to Eurocontrol fur support on development of 'Standardized European Rules of the Air' (SERA)

\section{Integrated Communications Navigation and Surveillance (ICNS) Conference}

April 10-12, 2018 